\title{
Effect of verapamil on systolic and diastolic coronary blood flow velocity in asymptomatic and mildly symptomatic patients with hypertrophic cardiomyopathy
}

\author{
P Petkow Dimitrow, M Krzanowski, R Niżankowski, A Szczeklik, J S Dubiel
}

\begin{abstract}
Objective-To assess non-invasively the effect of verapamil treatment on coronary blood flow velocity in asymptomatic and mildly symptomatic patients with hypertrophic cardiomyopathy.

Design-High frequency transthoracic Doppler echocardiography was used to compare resting phasic coronary blood flow velocity before and after a one month period of verapamil treatment in 17 patients (14 men and three women) with non-obstructive hypertrophic cardiomyopathy. Eighteen healthy subjects formed an age and sex matched control group. Systolic and diastolic coronary blood flow velocity was measured in the distal portion of left anterior descending coronary artery using high frequency transthoracic Doppler echocardiography. Blood flow velocity before and after verapamil was compared in the patients with cardiomyopathy and with the results in the control group.

Results-Compared with the controls, patients with hypertrophic cardiomyopathy had increased diastolic coronary blood flow velocity $(41.8(8.1) v 59.9(21.9) \mathrm{cm} / \mathrm{s}, \mathrm{p}<0.01)$ and a lower mean systolic coronary blood flow velocity $(18.7(10.8) v-11.2(27.5) \mathrm{cm} / \mathrm{s}, \mathrm{p}<0.01)$ before verapamil treatment. A backward pattern of systolic flow, manifested by negative values of coronary blood flow velocity, was recorded in eight of the patients, while no negative values were found in the controls. After verapamil treatment the retrograde systolic blood flow was restored to an anterograde pattern in only one patient. The mean value of systolic coronary blood flow velocity did not change significantly and remained lower than the systolic forward flow velocity in the controls $(-3.6(31.8) v 18.7(10.8) \mathrm{cm} / \mathrm{s}, \mathrm{p}<0.05)$. However, diastolic coronary blood flow velocity decreased significantly after verapamil $(59.9$ (21.9) $v 50.7(19.5) \mathrm{cm} / \mathrm{s} \mathrm{p}<0.05)$, reaching a level comparable with that in the controls $(50.7(19.5) v 41.8(8.1) \mathrm{cm} / \mathrm{s}, \mathrm{p}>0.05)$.

Conclusions-In contrast to healthy subjects, in non-obstructive hypertrophic cardiomyopathy the systolic pattern of coronary blood flow was heterogeneous (both retrograde and anterograde), and diastolic coronary blood flow velocity was abnormally increased, despite a lack of significant symptoms. Verapamil treatment did not restore the forward pattern of systolic blood flow but decreased diastolic blood flow velocity to a level comparable with that in healthy subjects.

(Heart 2000;83:262-266)
\end{abstract}

Keywords: hypertrophic cardiomyopathy; verapamil; coronary blood flow velocity

It has recently become possible to measure coronary blood flow velocity in the left anterior descending coronary artery using transthoracic $^{1-2}$ or transoesophageal ${ }^{3-5}$ Doppler echocardiography in patients with hypertrophic cardiomyopathy. With respect to systolic blood flow velocity, both negative (pathological) and positive (physiological) values have been recorded, representing pathological backward flow and physiological forward flow, respectively. ${ }^{134}$ For diastolic blood flow velocity there have been equivocal results (either increased or normal in comparison with healthy subjects). ${ }^{1-4}$ Such interstudy differences may have resulted from the effects of drug treatment, from varying symptom status, and from the magnitude of the left ventricular outflow tract gradient. We suspect that such diversity of coronary blood flow velocity values probably reflects the heterogeneity of patients with hypertrophic cardiomyopathy.

Our aim in the present study was to assess the pattern of coronary blood flow in a more homogeneous group of patients (that is, asymptomatic or mildly symptomatic and without left ventricular outflow tract obstruction), and to evaluate the effect of verapamil on systolic and diastolic coronary blood flow velocity. We also compared the measurements made in the patients with values in healthy subjects.

\section{Methods}

STUDY POPULATION

We studied 21 consecutive, asymptomatic or mildly symptomatic patients with nonobstructive hypertrophic cardiomyopathy. In 17 of these (14 men and three women, mean (SD) age 32 (6) years), a satisfactory recording of coronary blood flow velocity in the distal portion of the left anterior descending coronary artery was obtained on transthoracic Doppler echocardiography. Diagnosis of hypertrophic cardiomyopathy was based on typical clinical, ECG, and echocardiographic features. Ventricular septal thickness was markedly increased (to 23 (5) $\mathrm{mm}$ ), whereas posterior wall thickness was within the normal range (10 
(3) $\mathrm{mm}$ ), showing an asymmetric pattern of left ventricular hypertrophy (septum to posterior wall thickness ratio, $2.4(0.6))$. None of the patients had a left ventricular outflow tract gradient on Doppler echocardiography under resting conditions. A gradient of $>30 \mathrm{~mm} \mathrm{Hg}$ was induced in four patients by provocative tests.

No patients presented with hypercholesterolaemia, hypertension, or diabetes mellitus, and none smoked. Twelve patients were completely asymptomatic and the remaining five had only minor and transient symptoms - that is, dyspnoea on severe exertion. Untreated patients were assessed before and after one month of treatment with verapamil (240-360 mg daily).

The control group consisted of 22 healthy, age and sex matched subjects without any risk factors known to be associated with endothelial dysfunction (exclusion criteria identical to the hypertrophic cardiomyopathy group). Eighteen of these (14 men and four women, mean age 30 (5) years) had a satisfactory recording of coronary blood flow velocity in the distal portion of the left anterior descending coronary artery on transthoracic Doppler echocardiography.

All patients and controls gave informed consent for participation in the study.

\section{STUDY PROTOCOI}

Echocardiography of the left anterior descending coronary artery was performed using the Sequoia 512 ultrasound apparatus (Acuson, Mountain View, California, USA), with multifrequency transducer allowing on-line change of frequency separately in cross sectional imaging (3.5-5.0 MHz), pulse Doppler (3.5-5.0 $\mathrm{MHz}$ ), and colour Doppler (2.5-3.5 MHz). This system provides an extremely wide dynamic range and preserves both phase and amplitude data, with a high spatial and temporal resolution.

The echocardiographic technique for assessment of the left anterior descending coronary artery was as described previously. ${ }^{1}$ Briefly, the patients were examined in the left lateral position using a modified left parasternal window. The ultrasound beam was angled laterally and superiorly to identify the anterior intraventricular sulcus. In this area, coronary blood flow in the distal portion of left anterior descending coronary artery was identified, guided by colour Doppler flow mapping. With a sample volume positioned on the colour signal in the artery, Doppler spectral tracings of flow velocity within the vessel lumen were recorded. The long axis sections were carefully

Table 1 Comparison of heart rate, systolic blood pressure, and double product in patients with hypertrophic cardiomyopathy (HCM) before and after verapamil treatment and in controls

\begin{tabular}{lccc}
\hline & $\begin{array}{l}\text { HCM } \\
\text { pre-verapamil }\end{array}$ & $\begin{array}{l}\text { HCM } \\
\text { post-verapamil }\end{array}$ & Controls \\
\hline Heart rate (beats/min) & $68(9)^{\star}$ & $64(5)$ & $72(9) \dagger$ \\
Systolic blood pressure (mm Hg) & $132(12)^{\star}$ & $121(10)$ & $126(8)$ \\
Double product & $9011(1644)^{\star}$ & $7755(932)$ & $8933(1079) \dagger$ \\
\hline
\end{tabular}

Values are mean (SD).

${ }^{\star} \mathrm{p}<0.05, \mathrm{HCM}$ pre-verapamil $v$ HCM post-verapamil; $t \mathrm{p}<0.05$, controls $v \mathrm{HCM}$ post-verapamil. adjusted to minimise the angle (which was kept below $60^{\circ}$ ) between the Doppler beam and the long axis of the artery and to ensure that sampling volume was located within the vessel lumen for as much of the cardiac cycle as possible. All studies of the Doppler signal and the cross sectional echocardiogram were recorded on half inch $(1.25 \mathrm{~cm})$ super-VHS videotape for off-line analysis. In the repeat study after verapamil treatment, special care was taken to examine the same portion of the artery and to obtain the same angle between the Doppler beam and direction of blood flow as in the initial examination $\left(42(3)^{\circ} v 40(2)^{\circ}, \mathrm{p}>0.05\right)$. This mean angle was also comparable between the cardiomyopathy patients and the controls $\left(42(3)^{\circ} v 44(6)^{\circ}, \mathrm{p}>0.05\right)$.

Four variables were measured by tracing the contour of the Doppler coronary blood flow velocity pattern: peak systolic velocity, peak diastolic velocity, systolic velocity-time integral, and diastolic velocity-time integral. Values for each variable were obtained by averaging measurements from five to seven cardiac cycles. Values before and after verapamil treatment were compared; comparison was also made between velocities recorded in the cardiomyopathy patients and the healthy controls.

At the time of echocardiographic assessment, heart rate and systemic blood pressure were measured. The double product, reflecting myocardial oxygen demand, was calculated as systolic blood pressure $\times$ heart rate in the controls and in the patients before and after verapamil treatment.

\section{STATISTICAL ANALYSIS}

Data are expressed as mean (SD). Comparisons of data within and between groups were done using the two sided Student's $t$ test for dependent and independent data, respectively. Correlation coefficients $(r)$ for regression analysis between coronary blood flow velocity indices and septal thickness were calculated. A $\mathrm{p}$ value $<0.05$ was considered significant.

\section{Results}

Verapamil treatment in the cardiomyopathy patients did not change septal thickness (23 (5) v 22 (5) $\mathrm{mm}, \mathrm{p}>0.05$ ) but significantly increased the left ventricular end diastolic dimension (42 (4) $v 45$ (5) mm, $\mathrm{p}<0.05$ ).

The heart rate, systolic blood pressure, and double product were comparable in the hypertrophic cardiomyopathy patients before verapamil and the healthy controls (table 1). Verapamil treatment significantly decreased heart rate, systolic blood pressure, and the double product in the patients (table 1).

Systolic coronary blood flow was detected in 15 of the patients and in all the controls. Before verapamil treatment, eight cardiomyopathy patients had a reversed (that is, backward) systolic coronary blood flow. Consequently, mean values of systolic coronary blood flow velocity and velocity-time integral were negative and significantly lower than in the control group (table 2). After verapamil treatment, the retrograde systolic coronary blood flow was 
Table 2 Comparison of coronary haemodynamic data in patients with hypertrophic cardiomyopathy (HCM) before and after verapamil treatment, and in controls

\begin{tabular}{lclc}
\hline & HCM pre-verapamil & HCM post-verapamil & Controls \\
\hline Peak sCBFV $(\mathrm{cm} / \mathrm{s})$ & $-11.2(27.5) \ddagger$ & $-3.6(31.8) \dagger$ & $18.7(10.8)$ \\
sVTI $(\mathrm{cm})$ & $-2.2(4.9) \ddagger$ & $-1.2(5.2) \dagger$ & $2.7(1.7)$ \\
Peak dCBFV $(\mathrm{cm} / \mathrm{s})$ & $59.9(21.9)^{\star} \ddagger$ & $50.7(19.5)$ & $41.8(8.1)$ \\
dVTI $(\mathrm{cm})$ & $21.9(10.1)^{\star} \ddagger$ & $17.1(8.1)$ & $13.6(2.6)$ \\
\hline
\end{tabular}

Value are mean (SD).

${ }^{\star} \mathrm{p}<0.05 \mathrm{HCM}$ pre-verapamil $v$ HCM post-verapamil; $\mathrm{tp}<0.05 \mathrm{HCM}$ post-verapamil $v$ controls; $\neq \mathrm{p}<0.01 \mathrm{HCM}$ pre-verapamil $v$ controls.

$\mathrm{dCBFV}$, diastolic coronary blood flow velocity; dVTI, diastolic velocity-time integral; HCM, hypertrophic cardiomyopathy; sCBFV, systolic coronary blood flow velocity; sVTI, systolic velocity-time integral.
Despite a lack of significant symptoms or left ventricular outflow tract gradient, systolic coronary blood flow was abnormally retrograde in eight of our patients before verapamil treatment. Restoration of the physiological, anterograde pattern of systolic coronary blood flow occurred only in one patient with verapamil treatment. In previous non-invasive ${ }^{134}$ and invasive $^{6-8}$ studies, a wide spectrum from negative to positive values of systolic coronary blood flow velocity was present in a heterogeneous population of patients with hypertrophic cardiomyopathy (that is, differing in symptom status and presenting with obstructive or nonobstructive disease). The effect of treatment on coronary blood flow pattern has not been yet been evaluated.

In hypertrophic cardiomyopathy, resting coronary blood flow within the left anterior descending coronary artery supplying the thickened septum is augmented. ${ }^{7910}$ The blood flow in this artery exceeds coronary blood flow in the circumflex artery supplying non-hypertrophied left ventricular wall by $30 \% .^{7}$ This finding is presumably related to higher basal myocardial oxygen requirements owing in part to the increased septal mass. ${ }^{710}$ The increased blood flow causes increased flow velocity when there is inadequate enlargement of left anterior descending coronary artery size to match the increased septal thickness. ${ }^{11}$ Such a mismatch has been reported in hypertrophic cardiomyopathy, ${ }^{12}$ where the left anterior descending coronary artery diameter was undersized in relation to the massively hypertrophied septum. The velocity-time integral of diastolic coronary blood flow velocity before verapamil treatment in our patients correlated with septal thickness, as in a previous study. ${ }^{2}$ This relation is in line with the concept that an increase in compensatory coronary blood flow matching a higher myocardial oxygen demand is partially dependent on septal hypertrophy. After verapamil, diastolic coronary blood flow velocity decreased but septal thickness remained unchanged, so this correlation became non-significant. We propose that verapamil decreases coronary blood flow velocity not by reducing septal thickness but by a fall in myocardial oxygen demand (reflected in a reduction in the double was heterogeneous in our group of patients.

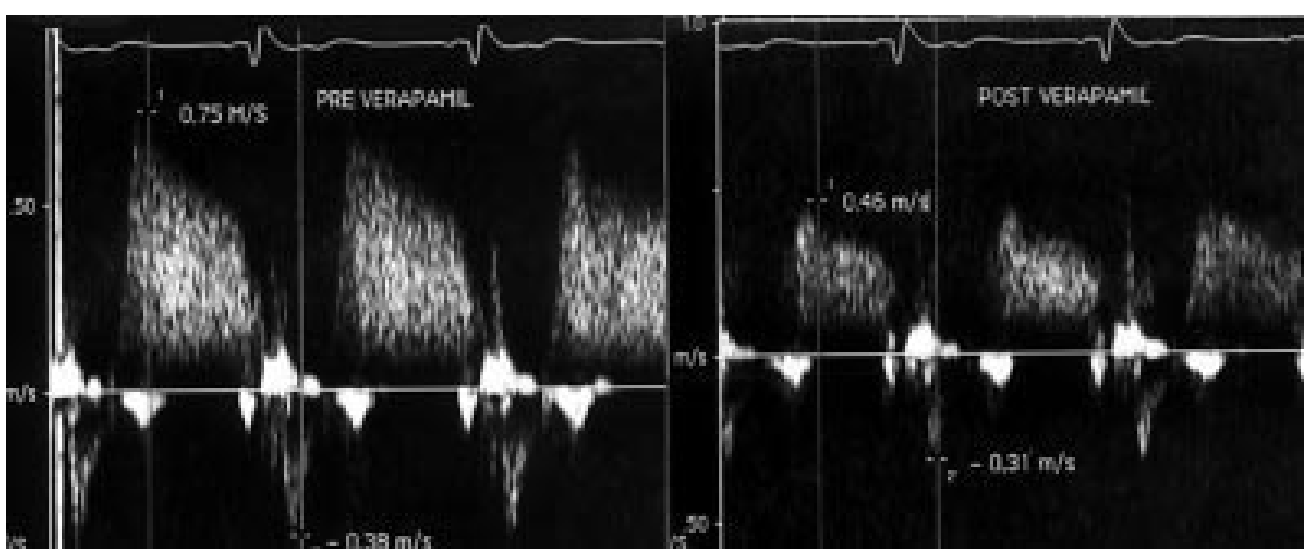

Figure 1 Example of coronary blood flow velocity (CBFV) changes. Doppler spectral velocity profiles pre-verapamil (left trace) and post-verapamil (right trace). Diastolic CBFV decreased from 0.75 to $0.46 \mathrm{~m} / \mathrm{s}$. Reversed systolic CBFV changed only slightly, from -0.38 to $-0.31 \mathrm{~m} / \mathrm{s}$. 
product). This suggestion received support from the propranolol induced reduction in myocardial oxygen demand and coronary blood flow in patients with obstructive hypertrophic cardiomyopathy ${ }^{13}$; in symptom-free patients, propranolol decreased the double product (by reducing left ventricular outflow tract gradient and heart rate) in parallel with a decrease in coronary blood flow. We suspect that in our patients the decrease in the double product may have been responsible for the decreased diastolic coronary blood flow velocity after verapamil. Diastolic coronary blood flow velocity was still higher (though not significantly so) than in the healthy controls, probably because of persisting massive septal hypertrophy. To clarify the statistical power of this difference a larger number of patients needs to be studied.

In the face of evidence that a lower resting diastolic coronary blood flow velocity is associated with greater exercise capacity in a previous study, ${ }^{5}$ the reduction in diastolic coronary blood flow velocity after verapamil in our patients can be regarded a favourable finding. It has recently been shown ${ }^{2}$ that reduced coronary flow reserve is caused by increased resting diastolic coronary blood flow velocity, whereas adenosine stimulated hyperaemic coronary blood flow velocity is comparable to normal values. Therefore interventions that decrease resting diastolic coronary blood flow velocity are likely to be useful. Further support for this hypothesis is provided by observations on patients with left ventricular hypertrophy secondary to hypertension or aortic stenosis. ${ }^{114}$ When symptoms are present in such patients, resting diastolic coronary blood flow velocity is nearly doubled in comparison with values in asymptomatic patients or controls. ${ }^{11}{ }^{14}$ Higher diastolic coronary blood flow velocity increases shear stress within the coronary arteries, and it has been postulated that prolonged augmentation of shear stress may impair coronary endothelial function in these patients and contribute to ischaemia. ${ }^{11}{ }^{15}$ In our patients, diastolic coronary blood flow velocity was increased before verapamil treatment even though the patients were asymptomatic and did not have an increased left ventricular systolic pressure. Therefore, a verapamil induced decrease in diastolic coronary blood flow velocity could, by reducing shear stress, diminish the coronary endothelial dysfunction that has previously been demonstrated in such patients. ${ }^{16}{ }^{17}$ Our results, along with data from previous studies, ${ }^{18-20}$ support the view that treatment should be given even in asymptomatic patients, particularly as abnormalities of myocardial metabolism similar to those found in ischaemic myocardium have been detected in such patients by 31phosphorus nuclear magnetic resonance spectroscopy. ${ }^{18}$ Verapamil has previously been used in asymptomatic and mildly symptomatic patients with hypertrophic cardiomyopathy with favourable effects. ${ }^{19} 20$ In these patients, verapamil reduced exercise induced scintigraphic perfusion defects ${ }^{19}$ and improved the left ventricular diastolic filling response to the cold pressor test. ${ }^{20}$ The improvement in these responses appeared to result from enhanced endothelium dependent coronary vasodilatation induced by verapamil - a phenomenon well documented in experimental studies. ${ }^{21-23}$

Previous studies ${ }^{1-4}$ using transthoracic and transoesophageal Doppler echocardiography showed discrepancies in diastolic coronary blood flow velocity values in patients with hypertrophic cardiomyopathy. These differences probably reflect the heterogeneous nature of the patient groups. Asami et al reported increased resting diastolic coronary blood flow velocity, ${ }^{2}$ as we did. Crowley et al found comparable diastolic coronary blood flow velocity measurements between healthy controls and patients with hypertrophic cardiomyopathy, independent of symptoms or left ventricular outflow tract obstruction ${ }^{1}$; however, information about the treatments used was not provided. Crowley's diastolic coronary blood flow velocity values in healthy subjects were closely comparable to our own. In other studies using transoesophageal echocardiography, patients with non-obstructive cardiomyopathy had similar mean diastolic coronary blood flow velocity to controls, ${ }^{3}$ whereas untreated patients with obstructive cardiomyopathy had increased diastolic coronary blood flow velocity. ${ }^{4} \mathrm{~A}$ similar discrepancy in the diastolic coronary blood flow velocity values has been reported in invasive studies. ${ }^{6924}$ In two studies, ${ }^{924}$ increased coronary blood flow velocity was found, whereas in others, ${ }^{68}$ the values were normal. Such discrepancies may reflect differences in myocardial oxygen demand, which is determined by multiple factors such as systolic left ventricular pressure (depending on the magnitude of the left ventricular outflow tract gradient), heart rate, septal thickness, and so on.

STUDY LIMITATIONS

Although the left anterior descending coronary artery was detected using combined Doppler and cross sectional imaging, the images were not of sufficient clarity to allow accurate measurement of small changes in vessel diameter. Therefore we limited our measurement to coronary blood flow velocity. Echo contrast agents have recently been introduced..$^{25}$ The increase in signal to noise ratio in vascular districts after intravenous injection of such agents should allow more complete imaging of the coronary artery. Without estimating coronary artery diameter, we were only able to measure changes in coronary blood flow velocity, not in coronary blood flow. However, it has been shown that changes in flow velocity closely reflect changes in flow, ${ }^{26}$ and coronary blood flow velocity has previously been used as a surrogate for coronary blood flow. ${ }^{1-5} 25$

Another limitation is the feasibility of transthoracic coronary blood flow velocity measurement. In our consecutive series of 21 patients, satisfactory measurements of coronary blood flow velocity were possible in 17 patients with respect to diastolic flow, and in 15 for systolic flow. Our inability to detect systolic coronary blood flow in two patients may have been the result of a very low systolic coronary blood flow 
velocity, which is not infrequently found in patients with hypertrophic cardiomyopathy using more precise invasive measurements. ${ }^{6}$ The success rate of our measurements was $81 \%$ ( $71 \%$ for systolic coronary blood flow), which is comparable to the detection rate of $71 \%$ achieved in a previous study. ${ }^{1}$ This limitation seems to be resolved by the use of echo contrast agents enhancing Doppler signal intensity. ${ }^{25}$

Regarding the accuracy of transthoracic Doppler measurement of coronary blood flow velocity, comparison with simultaneously performed intracoronary measurement has shown a highly satisfactory correlation. ${ }^{27}$

\section{CONCLUSIONS}

Systolic coronary blood flow was heterogeneous (both retrograde and anterograde) and diastolic coronary blood flow velocity was abnormally increased, despite the lack of significant symptoms, in our patients with non-obstructive hypertrophic cardiomyopathy. Verapamil treatment was generally unable to restore the forward pattern of systolic coronary blood flow, whereas it decreased diastolic coronary blood flow velocity to a level comparable to that found in healthy subjects. Further studies investigating the effect of verapamil or $\beta$ blocker treatment on the coronary flow dynamics in symptomatic patients are required. In addition, endothelium dependent and endothelium independent vasodilatation and their relation to the inducibility of ischaemia should be studied under various modes of treatment.

1 Crowley JJ, Dardas PS, Harcombe AA, et al. Transthoracic Doppler echocardiographic analysis of phasic coronary blood flow velocity in hypertrophic cardiomyopathy. Heart 1997;77:558-63.

2 Asami Y, Yoshida K, Hozumi T, et al. Assessment of coronary flow reserve in patients with hypertrophic cardiomyopathy using transthoracic color Doppler echocardioopathy using transthoracic colo
graphy. 7 Cardiol $1998 ; 32: 247-52$.

3 Tomochika Y, Tanaka N, Wasaki Y, et al. Assessment of flow profile of left anterior descending coronary artery in hypertrophic cardiomyopathy by transesophageal pulsed Doppler echocardiography. Am f Cardiol 1993;72:1425-30.

4 Memmola C, Iliceto S, Napoli VF, et al. Coronary flow dynamics and reserve assessed by transesophageal echocardiography in obstructive hypertrophic cardiomyopathy. Am $\mathcal{F}$ Cardiol 1994;74:1147-51.

5 Petkow Dimitrow P, Krzanowski M, Bodzon W, et al. Coronary flow reserve and exercise capacity in hypertrophic cardiomyopathy. Heart Vessels 1996;11:160-4.

6 Akasaka T, Yoshikawa J, Yoshida K, et al. Phasic coronary flow characteristics in patients with hypertrophic cardiomyopathy: a study by coronary Doppler catheter. $\mathcal{F}$ Am Soc Echocardiogr 1994;7:9-19.

7 Kyriakidis MK, Dernellis JM, Androulakis AE, et al. Changes in phasic coronary blood flow velocity profile and relative coronary flow reserve in patients with hypertrophic bstructive cardiomyopathy. Circulation 1997;96:834-41.

8 Higano ST, Yeo TC, Nishimura RA, et al. Coronary hemodynamics in hypertrophic cardiomyopathy: Intracoronary Doppler observations [abstract]. $\mathcal{F}$ Am Coll Cardiol 1997;29(suppl):126A.

9 Tsukahara R, Muramatu T, Akimoto N, et al. Evaluation of coronary flow characteristics in patients with hypertrophic cardiomyopathy using a Doppler guidewire [abstract]. $\mathcal{7}$ Am Coll Cardiol 1997;29(suppl):462A

10 Cannon RO, Rosing DR, Maron BJ, et al. Myocardial ischemia in patients with hypertrophic cardiomyopathy: contribution of inadequate vasodilator reserve and elevated left ventricular filling pressures. Circulation 1985;71:23443.

11 Isaaz K, Bruntz JF, Paris D, et al. Abnormal coronary flow velocity pattern in patients with ventricular hypertrophy, angina pectoris, and normal coronary arteries: a transesophageal Doppler echocardiographic study. Am f Heart
1994;128:500-10.

12 Kimball BP, LiPreti V, Bui S, et al. Comparison of proximal left anterior descending and circumflex coronary artery dimensions in aortic valve stenosis and hypertrophic cardiomyopathy. Am f Cardiol 1990;65:767-71.

13 Cuccurullo F, Mezzetti A, Lapenna D, et al. Mechanism of isoproterenol-induced angina pectoris in patients with obstructive hypertrophic cardiomyopathy and normal coronary arteries. Am f Cardiol 1987;60:667-73.

14 Oram H, Fehske W. Relationship between symptoms and coronary blood flow velocity in patients with aortic stenosis [abstract]. Circulation 1994;90(suppl):I-116.

15 O'Keefe JH, Owen RM, Bove AA. Influence of left ventricular mass on coronary cross-sectional area. Am 7 Cardiol 1987;59:1395-7.

16 Kodama K, Hamada M, Kazatani Y, et al. Clinical characteristics in Japanese patients with coexistent hypertrophic cardiomyopathy and coronary vasospasm. Angiolggy 1998;49:849-55.

17 Iida $\mathrm{H}$, Fujii T, Miura T, et al. Assessment of endothelium dependent coronary vasodilation in hypertrophic cardiomyopathy [abstract]. Circulation 1996;94:I-502.

18 Jung WI, Sieverding L, Breuer J, et al. 31P NMR spectroscopy detects metabolic abnormalities in asymptomatic patients with hypertrophic cardiomyopathy. Circulation 1998;97:2536-42.

19 Udelson JE, Bonow RO, O'Gara PT, et al. Verapamil prevents silent myocardial perfusion abnormalities during exercise in asymptomatic patients with hypertrophic cardiomyopathy. Circulation 1989;79:1052-60.

20 Petkow Dimitrow P, Surdacki A, Dubiel JS. Verapamil normalizes the response of left ventricular early diastolic filling to cold pressor test in asymptomatic and mildly symptomatic patients with hypertrophic cardiomyopathy. Cardiovasc Drugs Ther 1997;11:741-6.

21 Takase H, Moreau P, Kung Ch, et al. Antihypertensive therapy prevents endothelial dysfunction in chronic nitric oxide deficiency; effect of verapamil and trandolapril. Hypertension 1996;27:25-31.

22 Dagenais F, Cartier R, Hollmann C, et al. Calcium-channel blockers preserve endothelial reactivity after ischemiareperfusion. Ann Thorac Surg 1997;63:1050-6.

23 Dohi Y, Criscione L, Pfeiffer K, et al. Angiotensin blockade or calcium antagonists improve endothelial dysfunction in hypertension: studies in perfused mesenteric resistance hypertension: studies in perfused mesenteric

24 Krams R, Kofflard MJ, Duncker DJ, et al. Decreased coronary flow reserve in hypertrophic cardiomyopathy is related to remodeling of the coronary microcirculation. Circulation 1998;97:230-3.

25 Caiati C, Montaldo C, Zedda N, et al. New noninvasive method for coronary flow reserve assessment: contrastenhanced transthoracic second harmonic echo Doppler. Circulation 1999;99:771-8.

26 Wilson RF, Laughlin DE, Ackell PH, et al. Transluminal subselective measurement of coronary artery flow velocity and vasodilator reserve in man. Circulation 1985;72:82-92.

27 Bartel T, Baumgart S, Muller S, et al. Validation of transthoracic Doppler measurements of coronary flow velocity by intracoronary Doppler guidewire [abstract]. 7 Am Coll Cardiol 1998;31 (suppl C):243C. 